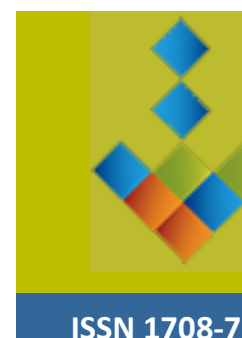

\title{
FADIO, un modèle inspirant pour le partage d'expertise
}

\section{FADIO: An Innovative Initiative to Share Expertise in Distance Education}

https://doi.org/10.18162/ritpu-2020-v17n1-15

Décrire FADIO est un défi pour moi tellement il y a de choses à dire sur l'ensemble de ses actions et réalisations. FADIO, c'est en premier lieu une initiative interrégionale portée par les établissements d'enseignement du Bas-Saint-Laurent et de la Gaspésie-Îles-de-la-Madeleine, qui ont fait le choix, en 2013, de travailler en collaboration pour favoriser le développement de la formation à distance et des pratiques de qualité.

Pour bien l'illustrer, je comparerais FADIO à une grande famille qui se donne un objectif commun où chaque membre est impliqué activement et est invité à contribuer à l'atteinte de cet objectif. Un de nos collaborateurs a utilisé récemment une expression qui nous décrit parfaitement bien : FADIO, c'est un écosystème éducatif vivant.

Chaque acteur éducatif est appelé à contribuer à sa façon au développement de bonnes pratiques reliées à la formation à distance. Que ce soit l'enseignant, le conseiller pédagogique, le technicien en informatique ou le gestionnaire, ces personnes jouent un rôle important dans la détermination des conditions gagnantes sur les plans pédagogique, technologique, technique et organisationnel de la formation à distance, et c'est pourquoi ils sont mis à contribution dans la réalisation du plan d'action de FADIO.

\section{FADIO depuis sa création}

Il y a quelques années, plusieurs facteurs auraient pu laisser croire que ce partenariat serait difficile à réaliser, car les réalités entre les ordres d'enseignement et entre les établissements étaient très différentes. Il y avait un écart important entre les établissements par rapport au développement de la formation à distance et une mise à niveau était nécessaire pour maximiser les retombées et faire en sorte que ça devienne un projet collectif. La persévérance et l'assiduité à la suite de quelques essais et erreurs permettent aujourd'hui de récolter les fruits du travail accompli et de bénéficier de retombées intéressantes. Mais comment tout cela a-t-il commencé?

1. Mylène Simard est coordonnatrice de FADIO. 
En 2008, les établissements d'enseignement du Bas-Saint-Laurent et de la Gaspésie-Îles-de-laMadeleine font face à une baisse démographique importante et au défi de plus en plus présent de trouver des solutions viables pour rendre accessible la formation. La formation à distance représente alors une avenue intéressante permettant de répondre à ce besoin. Après quelques cours à distance dispensés par les établissements, ces derniers comprennent vite que les coûts associés au développement sont relativement importants et qu'aucun d'entre eux ne dispose de ressources suffisantes pour assurer cette continuité.

À la suite de différents échanges sur le sujet et après avoir établi des paramètres de collaboration, ils forment la Table des partenaires et mettent sur pied le projet de Formation à distance interordres (FADIO). L'engagement est simple : s'impliquer activement, porter la vision de FADIO au sein de son établissement et partager son expertise. En 2013, ils signent leur première entente de collaboration, se dotent d'une structure de gouvernance collaborative, forment un comité de mise en œuvre et mettent en place un plan d'action collectif.

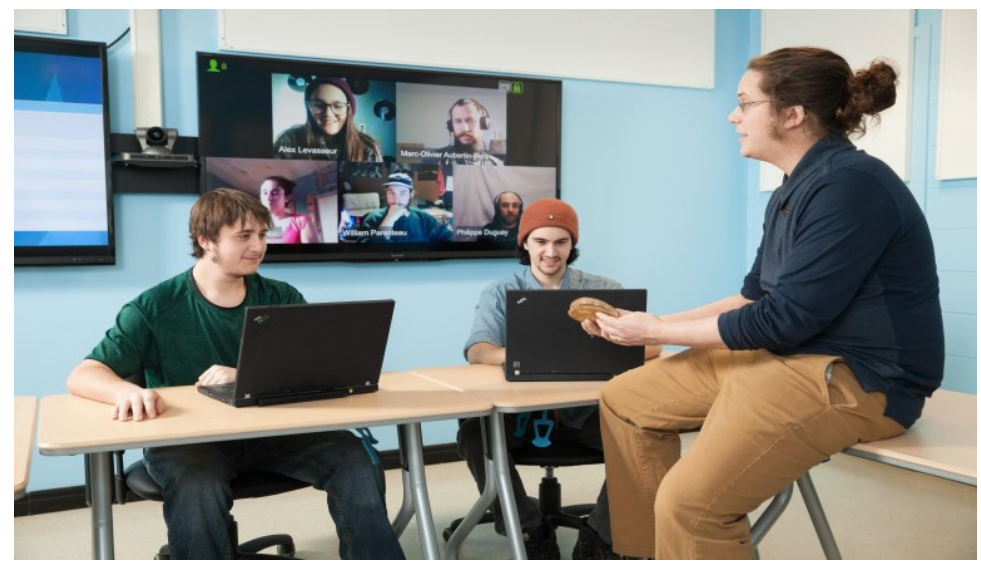

Figure 1

Source : Cégep de la Gaspésie et des îles

\section{Une gouvernance collaborative}

FADIO est une initiative unique qui s'inscrit parfaitement bien dans la mesure 22 du Plan d'action numérique en éducation et en enseignement supérieur, grâce à son modèle de gouvernance collaborative qui « favorise le partage d'expertise en formation à distance ».

Cette gouvernance collaborative fait en sorte qu'on adopte une approche basée sur les besoins et qu'on travaille à partir de dénominateurs communs afin d'impliquer tous les partenaires. Autre élément important, l'autonomie de chaque établissement est respectée, ce qui veut dire concrètement que chacun avance à son rythme. Dans une gestion de changement, il est impératif de ne mettre aucune pression et d'adopter plutôt une démarche d'accompagnement. Dans la réalisation des actions, on assure aussi une gestion optimale des ressources. Chaque formation développée ou service mis en place à FADIO est déployé par les personnes les mieux qualifiées pouvant y répondre, ce qui permet d'utiliser les compétences des personnes à leur plein potentiel. Enfin, pour optimiser la qualité dans les pratiques, on s'assure d'établir une cohésion entre la pédagogie, la technologie et la gestion dans chaque projet de formation à distance.

L'apport de FADIO au milieu de l'éducation est sa façon de mutualiser les ressources et de maximiser les retombées dans chaque établissement. Des communautés de pratique constituées de conseillers pédagogiques, de ressources enseignantes et de personnel en informatique partagent leur expertise et résolvent des problèmes. FADIO a aussi beaucoup misé sur le développement de la pédagogie en formation à distance. Nous faisons souvent l'erreur de nous 
attarder uniquement aux aspects technologiques et pourtant, la pédagogie doit demeurer au cœur de nos préoccupations. Autre action déployée par FADIO pour optimiser l'appui auprès des établissements, une équipe régionale de soutien a été mise sur pied par la création d'une ligne 1800 et d'une plateforme en ligne. Cette façon de travailler s'avère efficace et efficiente et permet à chaque établissement d'avoir accès en tout temps à des ressources compétentes. Enfin, pour rehausser la qualité des formations à distance et documenter les pratiques mises à l'essai sur le territoire, FADIO a instauré un réseau de praticiens-chercheurs interordres, ce qui stimule la recherche et l'expérimentation d'innovations en formation à distance. En plus de ces activités et services, d'autres initiatives sont également menées entre autres par la participation à des projets pilotes comme c'est le cas actuellement pour le projet de formation à distance à la formation générale des jeunes. Cinq commissions scolaires expérimentent et documentent différents modèles de formation à distance. Les résultats contribueront à l'avancement de l'une des mesures phares du Plan d'action numérique qui est de « favoriser le déploiement de la formation à distance à l'enseignement primaire et secondaire $»$.

En plus de toutes ces réalisations, toujours dans un esprit de partage d'expertise, nous organisons à FADIO trois événements d'envergure. D'abord, la Semaine de la formation à distance (FAD; FADIO, s.d.-c), un colloque en ligne offert gratuitement chaque hiver, donne l'occasion aux acteurs du milieu éducatif d'apprendre de bonnes pratiques et d'échanger avec des experts du milieu de l'éducation. À la dernière édition, près de 500 personnes en provenance du Québec, de la France, de la Belgique, de la Tunisie et du Maroc ont participé aux activités, montrant le leadership international de FADIO dans le domaine. Ensuite, la Rencontre des partenaires (FADIO, s.d.-b), un rassemblement bisannuel concentré sur deux jours, se veut un moment privilégié de discussion et de partage de pratiques pour tout le personnel des établissements d'enseignement associés à FADIO. Enfin, le Camp FAD (FADIO, s.d.-a), qui se tiendra pour la première fois l'automne prochain, sera une occasion de former et d'accompagner les ressources enseignantes du Québec dans la réalisation de leurs projets de FAD en étant épaulées par des experts dans le domaine.

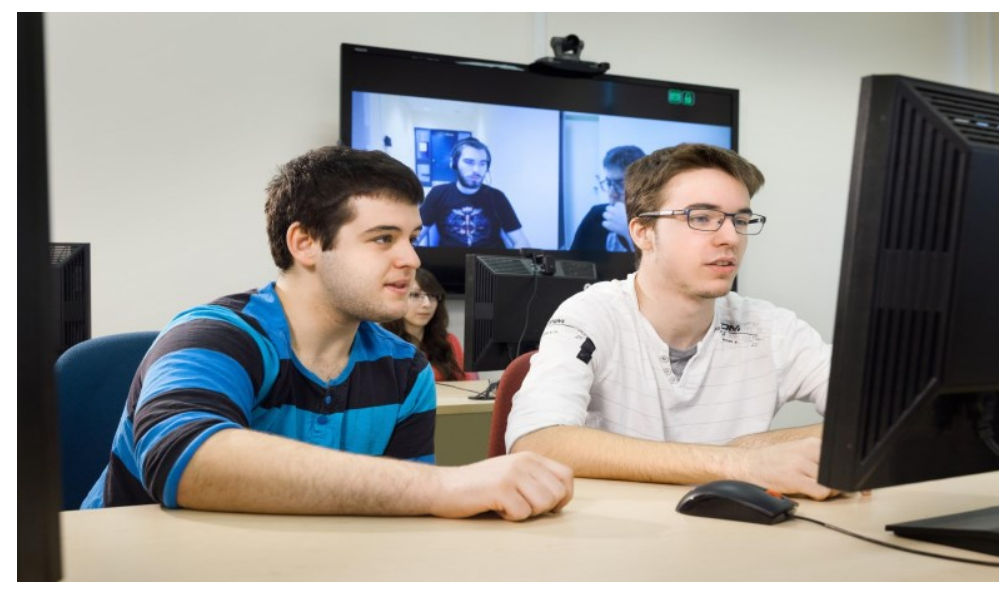

Figure 2

Source : Cégep de la Gaspésie et des îles

\section{Des retombées intéressantes}

Depuis sept ans, FADIO a mené à une offre de formations et de services variés permettant à une foule de professionnels de l'éducation de s'outiller en formation à distance. Les établissements n'ont jamais autant collaboré depuis sa création. On note une perception plus positive de la 
formation à distance dans le milieu et on a ainsi pu former des centaines de personnes pour les préparer à l'enseignement en ligne et à distance.

Le travail constant des partenaires a mené à la construction non seulement d'une gouvernance collaborative, mais d'un réseau fort en formation à distance qui donne accès à un large bassin d'expertise. Le travail de collaboration permet de proposer des solutions durables, de faire des choix technologiques judicieux, de regrouper des achats pour des services et de faire une économie de temps et d'argent.

Les établissements formant le regroupement FADIO ont accepté d'y partager plusieurs de leurs ressources qu'ils ont développé au cours des dernières années. Ainsi, autant les néophytes que les connaisseurs bénéficient de l'information et du soutien prodigués par le regroupement. L'esprit de partage qui les anime est à l'origine d'une panoplie de ressources d'une grande qualité accessibles non seulement aux membres de FADIO, mais également à l'ensemble du réseau éducatif québécois. Ceci répond d'ailleurs à une autre des mesures phares du Plan d'action numérique qui est de «développer une plateforme nationale de ressources éducatives numériques $»$.

La vision des partenaires pour rehausser la qualité de la formation à distance au Québec était de se doter d'un plan d'action permettant de démystifier la formation à distance, de mettre en valeur les pratiques gagnantes et de former et d'outiller le personnel enseignant, professionnel et de soutien ainsi que les gestionnaires.

La pertinence de FADIO et des actions qu'il suscite ressort à la lecture du rapport Demers, qui insiste sur l'importance de développer rapidement la formation à distance, notamment par l'entremise d'un consortium dont le but serait de « faciliter la collaboration entre établissements, de contribuer à la qualité de la formation offerte et de maximiser les ressources consacrées à la formation à distance » (Demers, 2014, p. 6). Il appert que FADIO tire pleinement profit de ces recommandations. En outre, l'association interordres qu'il propose en fait un maillon fort du développement des nouvelles compétences numériques, une mesure centrale du Plan d'action numérique en éducation et en enseignement supérieur (Ministère de l'Éducation et de l'Enseignement supérieur, 2018). Effectivement, cette mesure, tout comme le regroupement FADIO, touche tous les ordres d'enseignement, du primaire à l'université en passant par la formation des adultes et la formation professionnelle. Bref, FADIO fait avancer la formation à distance au Québec par les multiples occasions d'initiation, de formation, d'innovation et de partage qu'il crée. La communauté que les dix-huit partenaires ont ainsi générée a permis de réaliser beaucoup plus que ce que chacun de ses membres aurait accompli individuellement.

\section{Références}

Demers, G. (2014). Rapport final du chantier sur l'offre de formation collégiale. Ministère de l'Enseignement supérieur, de la Recherche et de la Science. https://eduq.info/xmlui/handle/11515/31972

FADIO (s.d.-a). Camp FAD. http://fadio.net/evenements/camp-fad

FADIO (s.d.-b). Rencontre des partenaires. http://fadio.net/evenements/rencontre-despartenaires

FADIO (s.d.-c). Semaine de la formation à distance. http://fadio.net/evenements/semaine-dela-formation-a-distance

Ministère de l'Éducation et de l'Enseignement supérieur. (2018). Plan d'action numérique en éducation et en enseignement supérieur. Les élèves et les étudiants : au cour de la révolution numérique. Gouvernement du Québec. http://education.gouv.qc.ca/... 